\title{
Radiation therapy: current and evolving strategies for nonmalignant intracranial tumors
}

\author{
Walter D. Johnson, M.D. \\ Department of Neurosurgery, Loma Linda University Medical Center, Loma Linda, California
}

$\mathrm{R}$ ADIATION, received at its invention as a great novelty, rapidly demonstrated that its unrestricted use translated into catastrophic complications, mostly in the form of cancers. Over time, limits were imposed to reduce exposure of personnel and surrounding body structures, yet deliver an appropriate dose to a specific target to achieve a particular goal. This is now achieved with modern forms of radiation by complex algorithms and highpowered computer technology. The paradox remains that this potentially harmful energy source may be harnessed and with careful planning be an effective instrument in the treatment of various tumors, yet may still have unwanted, delayed consequences.

In this month's topic of Neurosurgical Focus, we explore current radiation treatment of benign intracranial tumors. The first article is a review of the current management strategies of various benign tumors using state of the art radiation methods, highlight some of the differences in these techniques, and finally demonstrate how these can be complementary to the surgical treatment of these tumors. Gopalan and Lee explore the use of stereotactic radiosurgery (primarily Gamma Knife) and CyberKnife, respectively, in the treatment of craniopharyngioma. Pearson describes the evolution that treatment of atypical meningioma has undergone over the past several years with changing diagnostic criteria. Romanelli reviews radiation for hypothalamic hamartomas and Ghostin,e the treatment of pituitary adenomas.

Juxtaposed to these fine articles describing the benefits of radiation therapy is the excellent, yet sobering, contribution of Umansky documenting in graphic detail the consequence of radiation therapy, given in the past for relatively benign conditions. Although these radiation-induced tumors are, thankfully, not common in a typical neurosurgical practice, the consequences of even "benign" doses of radiation may, at some point in the future, result in unacceptable outcomes. Will we be seeing more of these tumors in the future as we refer more patients for radiation? This notion is highlighted by the critique and comments of Mathiesen.

This month we are provided with a real treat in the form of an in-depth editorial by Dr. Roberto Heros, describing how current radiation techniques have influenced his surgical attitude towards nonmalignant intracranial tumors. Dr. Heros candidly explains his approach to these various tumors, separating them by location and histology. Dr. Heros continues his distinguished career as a neurosurgeon and an educator by further educating us in his balanced and comprehensive approach to these challenging tumors. I am honored and extremely grateful to Dr. Heros for providing his thoughts for this journal.

Finally, I would like to thank Dr. Martin Weiss for his inspiration and expert guidance from the creation to the culmination of this topic. In addition, I wish to say a heartfelt "Thank you!" to Ms. Margie Shreve for her limitless patience and expertise provided to this novice editor throughout the production process. 